\begin{tabular}{|c|l|}
\hline Title & Photocurrent enhancement in light-soaked chal cogenide glasses \\
\hline Author(s) & Toyosawa, Naoko; Tanaka, Keiji \\
\hline Citation & $\begin{array}{l}\text { PHY SICAL REVIEW B, 56(12), 7416-7421 } \\
\text { https://doi.org/10.1103/PhysRevB.56.7416 }\end{array}$ \\
\hline Issue Date & 1997-09-15 \\
\hline Doc URL & http://hdl.handle.net/2115/5827 \\
\hline Rights & Copyright $\odot$ 1997 A merican Physical Society \\
\hline Type & article \\
\hline File Information & PRB56-12.pdf \\
\hline
\end{tabular}

Instructions for use 


\title{
Photocurrent enhancement in light-soaked chalcogenide glasses
}

\author{
Naoko Toyosawa and Keiji Tanaka* \\ Department of Applied Physics, Faculty of Engineering, Hokkaido University, Sapporo 060, Japan
}

(Received 24 February 1997)

\begin{abstract}
Photocurrent changes with light soaking have been studied in amorphous $\mathrm{As}_{2} \mathrm{~S}(\mathrm{Se}, \mathrm{Te})_{3}$ and $\mathrm{Se}$ and crystalline $\mathrm{As}_{2} \mathrm{~S}_{3}$ and $\mathrm{Se}$ at 10-300 K. In the chalcogenide glasses the photoconductive degradation occurs at $300 \mathrm{~K}$, while at low temperatures photocurrents in subband-gap spectral regions increase with light soaking. In the crystalline samples, no such changes are detected. The photoconductive enhancement appears to be related to the photoinduced midgap absorption, and the microscopic mechanism is discussed in a unified way. The observations are also compared with those in the Staebler-Wronski effect in amorphous Si:H films. [S0163-1829(97)04436-6]
\end{abstract}

\section{INTRODUCTION}

Effects of light soaking on dark conductivities and photoconductivities in amorphous semiconductors are important subjects from both fundamental and practical points of view. ${ }^{1,2}$ Specifically, the Staebler-Wronski effect appearing in hydrogenated amorphous $\mathrm{Si}(a-\mathrm{Si}: \mathrm{H})$ (Ref. 3) poses serious problems in solar-cell applications, and extensive studies are being performed. Also in chalcogenide glasses at room temperature, Shimakawa et al. have demonstrated a similar degradation phenomenon of photocurrents. ${ }^{4}$ These photodegradation phenomena are considered to be caused by a photoinduced creation of some kind of defects; the details are not elucidated. On the other hand, in previous studies, ${ }^{5,6}$ it has been demonstrated that photocurrents in amorphous Se and $\mathrm{As}_{2} \mathrm{~S}_{3}$ held at low temperatures increase with light soaking; i.e., photoconductive enhancement can occur. However, detailed characteristics of the photoconductive enhancement have not been investigated, and accordingly the mechanism remains to be studied.

In the present paper, therefore, extending the previous studies ${ }^{5,6}$ we will examine the details of the photocurrent increase in chalcogenide glasses. The present study will provide a guiding concept for modifying photoconductive characteristics in other amorphous materials such as $a-\mathrm{Si}: \mathrm{H}$ films.

\section{EXPERIMENT}

Samples investigated were amorphous (a-) $\mathrm{As}_{2} \mathrm{~S}_{3}$, $\mathrm{As}_{2} \mathrm{Se}_{3}, \mathrm{As}_{2} \mathrm{Te}_{3}$, and $\mathrm{Se}$, and crystalline (c-) $\mathrm{As}_{2} \mathrm{~S}_{3}$ and Se. For $a-\mathrm{As}_{2} \mathrm{~S}(\mathrm{Se}, \mathrm{Te})_{3}$, melt-quenched bulk glasses were employed. The flakes were polished to a thickness of $\sim 1 \mathrm{~mm}$ with a lateral dimension of $\sim 5 \mathrm{~mm}$ using alumina powders, and then these were annealed for $1 \mathrm{~h}$ at around the glasstransition temperatures ${ }^{7}$ in vacuum. For $a$-Se, thin films which were evaporated on to slide-glass substrates at room temperature were employed. ${ }^{8}$ Thickness of the films was $\sim 0.4 \mu \mathrm{m}$. For conductivity measurements of these amorphous samples, Au-film interdigital electrodes (20-pair fingers, $10 \mu \mathrm{m}$ gap separation, $1 \mathrm{~mm}$ gap width) were deposited by vacuum evaporation. Orpiment, $c-\mathrm{As}_{2} \mathrm{~S}_{3}$, was obtained from Yakut (Russia), and photoresponses for cleaved sur- faces ( $a-c$ crystalline plane) were monitored using Au-film coplanar electrodes $(100 \mu \mathrm{m}$ gap separation and $2 \mathrm{~mm}$ gap width). Trigonal Se films were obtained by thermal crystallization of $a$-Se, and similar coplanar electrodes were deposited. These samples were mounted in an optical cryostat, and the temperature was varied between 10 and $420 \mathrm{~K}$.

Light soaking and photocurrent measurements were performed using a $250 \mathrm{~W}$ ultrahigh-pressure $\mathrm{Hg}$ lamp, ${ }^{9}$ a $500 \mathrm{~W}$ Xe lamp, and an Ar laser. Light soaking was made with these sources, and photocurrents were simultaneously monitored under these excitations with applied dc biases of $0.1-50 \mathrm{~V}$ using a digital electrometer (Advantest, R8340A). The light intensity was held less than $50 \mathrm{~mW} / \mathrm{cm}^{2}$, unless otherwise specified, and accordingly the photoeffects ${ }^{2,10}$ and temperature rise induced by intense illumination could be neglected. In addition, photocurrent spectra before and after light soaking were probed using the Xe lamp, a conventional monochromator, and the electrometer. The wavelength resolution was $\sim 5 \mathrm{~nm}$, and the light intensity is less than $\sim 1 \mathrm{~mW} / \mathrm{cm}^{2}$. Optical absorption spectra were measured using a conventional spectrometer.

\section{RESULTS}

\section{A. Exposure-time dependence}

Figure 1 shows photocurrent changes for $a-\mathrm{As}_{2} \mathrm{~S}_{3}$ monitored under light soaking from the $\mathrm{Hg}$ lamp. At $300 \mathrm{~K}$, we see that the photocurrent decreases with illumination, that is the photoconductive degradation occurs. This feature is qualitatively consistent with those reported previously for annealed films. ${ }^{2,4,11}$ Also in agreement with previous observations, the degradation has appeared in $a-\mathrm{As}_{2} \mathrm{Se}_{3},{ }^{2,4,11}$ but not in $a$-Se (Ref. 2) and $a-\mathrm{As}_{2} \mathrm{Te}_{3}$ (Ref. 12). As shown in the figure, however, at $100 \mathrm{~K}$ the photocurrent increases with illumination from the $\mathrm{Hg}$ lamp. ${ }^{6}$ Similar photocurrent increases at low temperatures have been detected also in $\mathrm{As}_{2} \mathrm{Se}(\mathrm{Te})_{3}$ and $\mathrm{Se}$.

On the other hand, light-soaking effects upon dark currents could not be investigated in detail due to small signals. That is, in $\mathrm{As}_{2} \mathrm{~S}_{3}$ at temperatures below $300 \mathrm{~K}$, dark currents could not be measured (less than $0.1 \mathrm{pA}$ ). In $\mathrm{As}_{2} \mathrm{Se}(\mathrm{Te})_{3}$ and $\mathrm{Se}$, no appreciable changes in dark currents with light soak- 


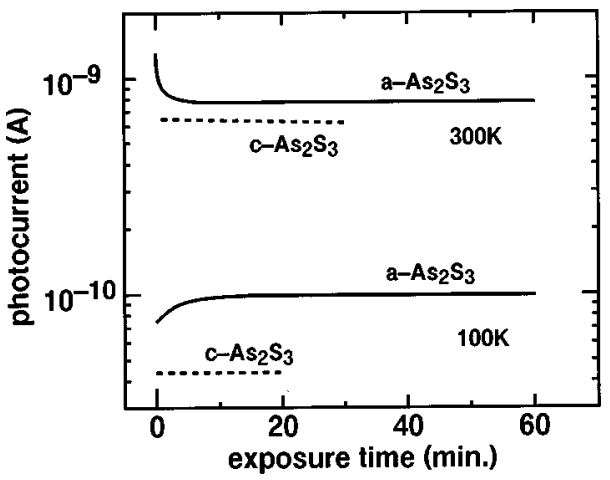

FIG. 1. Photocurrents in amorphous ( $a-) \mathrm{As}_{2} \mathrm{~S}_{3}$ (solid lines) and crystalline ( $c-) \mathrm{As}_{2} \mathrm{~S}_{3}$ (dotted lines) as a function of exposure time at 100 and $300 \mathrm{~K}$. The samples are illuminated with light $(50$ $\mathrm{mW} / \mathrm{cm}^{2}$ ) from an $\mathrm{Hg}$ lamp under bias voltages of $10 \mathrm{~V} / 10 \mu \mathrm{m}$ for $a-\mathrm{As}_{2} \mathrm{~S}_{3}$ and $50 \mathrm{~V} / 100 \mu \mathrm{m}$ for $c-\mathrm{As}_{2} \mathrm{~S}_{3}$.

ing were detected at room temperature, ${ }^{2}$ and at low temperatures dark currents could not be measured.

As shown in Fig. 1, in $c-\mathrm{As}_{2} \mathrm{~S}_{3}$ no light-soaking effect upon photocurrents appears. Neither has it appeared in $c$-Se. These observations suggest that the light-soaking effect is characteristic of the amorphous material.

Effects of light spectra and intensities upon the photocurrent enhancement have been studied, an example being shown previously. ${ }^{6}$ In more detail, when an $\mathrm{As}_{2} \mathrm{~S}_{3}$ glass was illuminated at $13-100 \mathrm{~K}$ with monochromatic light of 2.48$2.76 \mathrm{eV}$ with light intensity of $\sim 10 \mathrm{~mW} / \mathrm{cm}^{2}$, the photoconductive enhancement occurred qualitatively in the same way as that shown in Fig. 1 [and Fig. 3(a)]. In addition, a similar enhancement occurred more promptly when light with 2.54 $\mathrm{eV}$ was strengthened to $\sim 2 \mathrm{~W} / \mathrm{cm}^{2}$, while we might assume appreciable temperature rise under this condition.

\section{B. Temperature dependence}

Figure 2 shows temperature dependences of dark currents and photocurrents monitored at a photon energy of $2.8 \mathrm{eV}$ in $a-\mathrm{As}_{2} \mathrm{~S}_{3}$. The dark conductivity at $350 \mathrm{~K}$ is estimated roughly at $10^{-14} \mathrm{~S} / \mathrm{cm}$, and it is thermally activated with the activa-

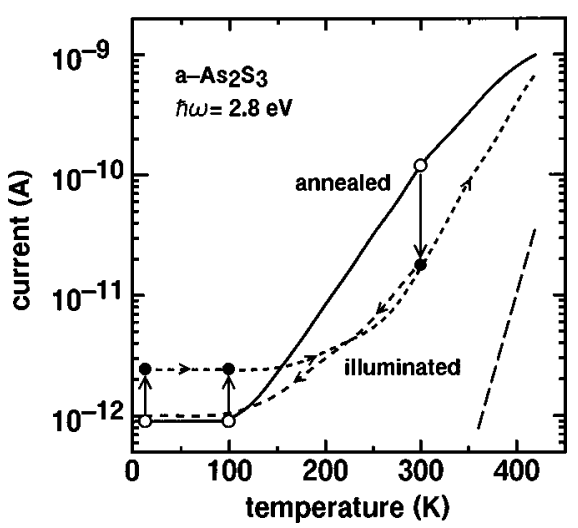

FIG. 2. Temperature dependence of dark current (dashed line) and photocurrents in the annealed (solid line) and light-soaked (dotted line) states in an $a-\mathrm{As}_{2} \mathrm{~S}_{3}$ sample. The photocurrents are probed with light of $2.8 \mathrm{eV}$ and $\sim 1 \mathrm{~mW} / \mathrm{cm}^{2}$. Solid arrows indicate the changes induced with light soaking ( $\mathrm{Hg}$ lamp for $30 \mathrm{~min}$ ). tion energy of $\sim 1.0 \mathrm{eV}$, which is comparable with previous results of $0.4-1.2 \mathrm{eV} .{ }^{13-17}$ The temperature dependence of the photocurrent in the annealed state is also consistent with a previous result. ${ }^{18}$ That is, at around $300 \mathrm{~K}$ the photocurrent increases with an activation energy of $0.2 \mathrm{eV}$, and at low temperatures the photocurrent becomes mostly constant.

Figure 2 also shows the light-soaking effects. We see that light soaking from the $\mathrm{Hg}$ lamp induces a decrease in the photocurrent at around $300 \mathrm{~K}$, while at temperatures below $150 \mathrm{~K}$ it gives rise to the photocurrent increase.

More interesting in Fig. 2 may be the photocurrent changes with temperature variations as follows. First, the annealed and the light-soaked states at $300 \mathrm{~K}$ give a similar photocurrent level when the sample is cooled down to temperatures below $100 \mathrm{~K}$. Second, the photoconductive enhanced state at low temperatures reduces to the photoconductive degraded state when the sample is heated to $300 \mathrm{~K}$, and then it recovers to the annealed state at temperatures below $100 \mathrm{~K}$. That is, the enhanced state disappears with annealing at $300 \mathrm{~K}$. In more detail, isochronal annealing investigations have demonstrated that a photoconductive enhancement at $\hbar \omega=1.4 \mathrm{eV}$ induced at $13 \mathrm{~K}$ by illumination from the $\mathrm{Hg}$ lamp disappears completely with annealing at $200 \mathrm{~K}$ for $1 \mathrm{~h}$. Third, the degraded state at $300 \mathrm{~K}$ recovers to the initial state only with annealing at the glass-transition temperature $\sim 450 \mathrm{~K}$ (Ref. 7) which is consistent with the previous observations. ${ }^{2,4}$

\section{Spectral dependence}

Figure 3(a) shows photocurrent spectra in $a-\mathrm{As}_{2} \mathrm{~S}_{3}$ before and after light soaking from the $\mathrm{Hg}$ lamp for $30 \mathrm{~min}$. The spectrum in the annealed state at $300 \mathrm{~K}$ is similar to those reported previously. ${ }^{19-21}$ The band-to-band excitation occurs at $\hbar \omega \geqslant 2.4 \mathrm{eV}$, which is consistent with the Tauc optical band gap energy $E_{g} .{ }^{1}$ We see that the light soaking at $300 \mathrm{~K}$ gives rise to the photocurrent decrease over the entire spectral region investigated. However, the photocurrent increase induced at $13 \mathrm{~K}$ is remarkable at the subband-gap spectral region $\left(\hbar \omega<E_{g}\right)$.

Photocurrent spectra in $a-\mathrm{As}_{2} \mathrm{Se}(\mathrm{Te})_{3}$ and $a$-Se before and after band-gap light soaking are also shown in Figs. 3(b), $3(\mathrm{c})$, and 3(d). At $300 \mathrm{~K}$, the photoconductive degradation appears in $a-\mathrm{As}_{2} \mathrm{Se}_{3}$ over the entire spectral range, while in $a-\mathrm{As}_{2} \mathrm{Te}_{3}$ and $a$-Se no appreciable changes appear. However, at $13 \mathrm{~K}$, the photoconductive enhancements occur in all the materials at subband-gap spectral regions.

Figure 4 compares the photocurrent and optical absorption spectra in $\mathrm{As}_{2} \mathrm{~S}_{3}$ at $13 \mathrm{~K}$ before and after light soaking. We see that before and after light soaking the photocurrent and the absorption coefficient manifest very similar spectra. In fact, the increases in the photocurrent and the absorption coefficient are quantitatively the same. The increase in the absorption spectra at low temperatures was originally discovered by Bishop et al., ${ }^{22}$ and is referred to as "photoinduced midgap absorption", since the absorption increase is prominent in the subband-gap spectral region.

In addition to the very similar spectra, the photoconductive enhancement has resembled the photoinduced absorption increase in two respects. ${ }^{2,22}$ First, the photoconductive enhancement in $\mathrm{As}_{2} \mathrm{~S}_{3}$ has disappeared with annealing at 


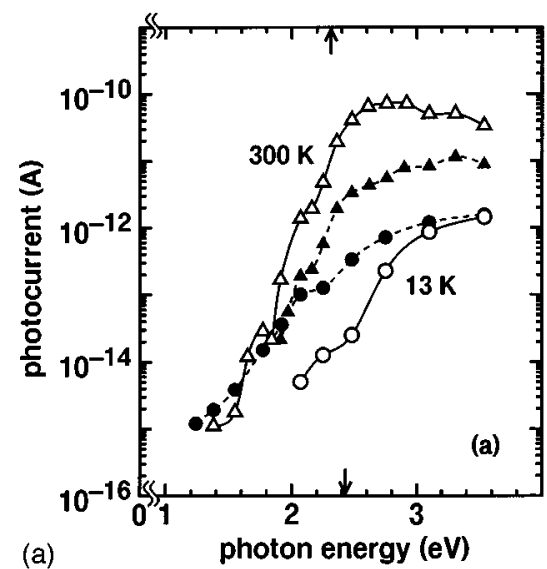

(a)

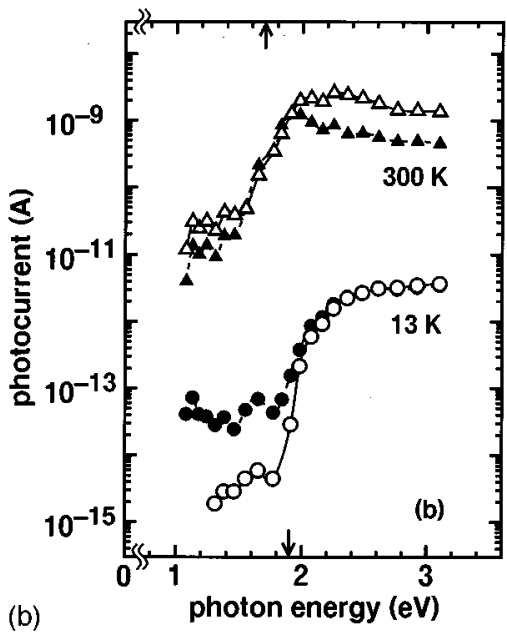

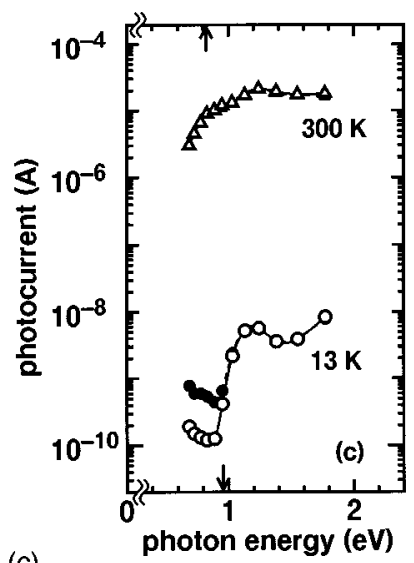

(c)

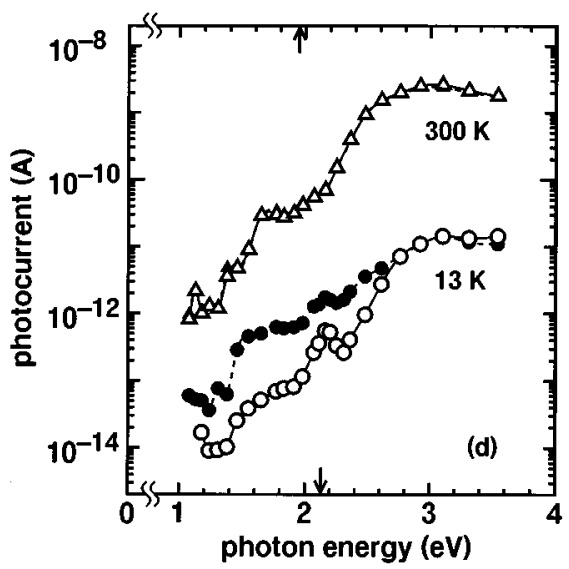

(d)

FIG. 3. Photocurrent spectra in (a) $a-\mathrm{As}_{2} \mathrm{~S}_{3}$, (b) $\mathrm{As}_{2} \mathrm{Se}_{3}$, (c) $\mathrm{As}_{2} \mathrm{Te}_{3}$, and (d) $a$-Se in annealed (open symbols with solid lines) and light-soaked states (solid symbols with dotted lines) at $13 \mathrm{~K}$ (circles) and $300 \mathrm{~K}$ (triangles). The photocurrents are normalized at a probe light intensity of $1 \mathrm{~mW} / \mathrm{cm}^{2}$ and an applied bias voltage of $1 \mathrm{~V} / 10 \mu \mathrm{m}$. Arrows at the bottom and the top indicate the Tauc optical bandgap energies at low temperatures (10-80 K) and $300 \mathrm{~K}$ (Refs. 1, 34, and 35).

200-300 K. Second, the photocurrent increase has been erased with illumination of subband-gap light. For instance, in $\mathrm{As}_{2} \mathrm{~S}_{3}$, the photoconductive enhancement has mostly disappeared with illumination of $1.4 \mathrm{eV}$ light.

\section{DISCUSSION}

Table I summarizes some observations. We see two important features. One is that the light-soaking effect is inher-

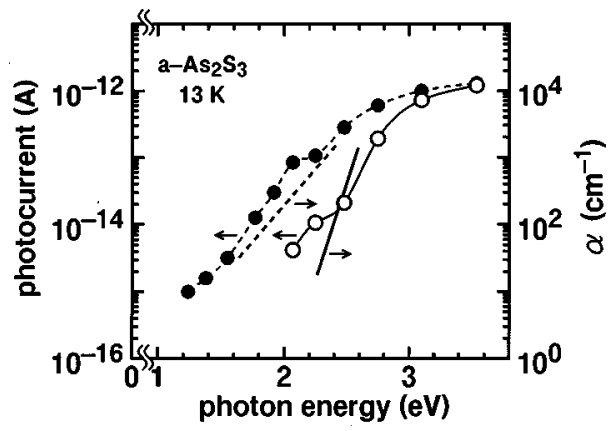

FIG. 4. Photocurrent spectra (circles with left-hand-side scale) and absorption-coefficient spectra (right-hand-side scale) in $a-\mathrm{As}_{2} \mathrm{~S}_{3}$ in the annealed state (solid lines) and the light-soaked state (dotted lines) at $13 \mathrm{~K}$. ent to the glasses. No changes appear in the crystalline materials. The other is that the photoconductive degradations evaluated at room temperature depend upon the kinds of glasses, while in marked contrast, the photoconductive enhancements at $13 \mathrm{~K}$ are roughly the same in all the amorphous materials investigated.

In addition, it has been demonstrated that the photoconductive enhancement is closely related with the photoinduced midgap absorption in spectrum (Fig. 4). We can, therefore, assume that the photocurrent increase is caused by the absorption increase, which can be responsible for the increase in the number of photoexcited carriers. Changes in the carrier mobility and the lifetime seem to provide little effects, since the photocurrent is mostly unaffected in the bandgap spectral regions (Fig. 3). Note that the present view is different from that by Johanson et al. ${ }^{5}$ who have assumed that the photoconductive enhancement is caused by the photodarkening phenomenon. ${ }^{2}$ However, the annealing kinetics cannot be understood with their model.

\section{A. Charged defect model}

A conventional way to understand the photoconductive changes in chalcogenide glasses may be to follow the socalled charged defect concept originally proposed by Mott 
TABLE I. Physical characteristics and photocurrent changes in the materials investigated in the present study. Listed are, from the left-hand to the right-hand side, the dark conductivity $\sigma_{d}$ at $300 \mathrm{~K}$, the photoconductivity $\sigma_{p}$ at $300 \mathrm{~K}$ under a band-gap illumination of 1 $\mathrm{mW} / \mathrm{cm}^{2}$, the photocurrent decrease at $300 \mathrm{~K} \Delta i_{p}(300 \mathrm{~K})$, the photocurrent increase in subband-gap spectral regions at $13 \mathrm{~K} \Delta i_{p}(13 \mathrm{~K})$, the energy heights of $E_{1}$ and $E_{2}$, the Tauc optical bandgap energy $E_{g}$ at $300 \mathrm{~K}$ (Refs. 1, 34, and 35), and the glass-transition temperature $T_{g}$ (Ref. 7).

\begin{tabular}{|c|c|c|c|c|c|c|c|c|}
\hline Material & $\sigma_{d}(\mathrm{~S} / \mathrm{cm})$ & $\sigma_{p}(\mathrm{~S} / \mathrm{cm})$ & $\Delta \sigma_{p}(300 \mathrm{~K})$ & $\Delta \sigma_{p}(13 \mathrm{~K})$ & $E_{1}(\mathrm{eV})$ & $E_{2}(\mathrm{eV})$ & $E_{g}(\mathrm{eV})$ & $T_{g}(\mathrm{~K})$ \\
\hline$a-\mathrm{As}_{2} \mathrm{~S}_{3}$ & $<10^{-15}$ & $10^{-12}$ & -1 order & +1 order & $0.3-0.5$ & $0.9-1.4$ & 2.4 & 470 \\
\hline$a-\mathrm{As}_{2} \mathrm{Se}_{3}$ & $10^{-12}$ & $10^{-10}$ & -0.5 order & +1 order & $0.1-0.7$ & $0.9-1.3$ & 1.7 & 460 \\
\hline$a-\mathrm{As}_{2} \mathrm{Te}_{3}$ & $10^{-6}$ & $10^{-6}$ & $\sim 0$ & +1 order & $0.1-0.7$ & $<0.7$ & 0.8 & 410 \\
\hline$a-\mathrm{Se}^{2}$ & $10^{-12}$ & $10^{-11}$ & $\sim 0$ & +1 order & $0.2-0.6$ & $<0.7$ & 2.0 & 310 \\
\hline$c-\mathrm{As}_{2} \mathrm{~S}_{3}$ & $<10^{-14}$ & $10^{-9}$ & 0 & 0 & & & 2.8 & \\
\hline$c-\mathrm{Se}$ & $10^{-8}$ & $10^{-5}$ & 0 & 0 & & & 1.8 & \\
\hline
\end{tabular}

et al. ${ }^{1,2}$ Otherwise, we may follow the valence-alternationpair model proposed by Kastner et al. ${ }^{1}$ Following these models, we here employ a configuration diagram illustrated in Fig. 5. In this diagram, it is essential that the energy minimum of the photoexcited state is located above the $D^{0}$ state. $^{23}$ That is, photoexcited states after lattice relaxation and $D^{0}$ possess similar atomic structures. The diagram is the same as the previous one, ${ }^{6}$ except that the energy barrier in the excited state is excluded here. ${ }^{24}$

As illustrated in Fig. 5, the photoconductive enhancement at low temperatures can be accounted for with the appearance of the $D^{0}$ states. ${ }^{6} D^{0}$ can be created from $D^{-}\left(\right.$and $D^{+}$) and a normal bonding structure by photoexcitation, and the defect can exist in stable at low temperatures since there exists the energy barrier of $E_{1}$.Here, this explanation is completely the same as that employed for the photoinduced midgap absorption. ${ }^{1}$ The $D^{0}$ can be a source of the photoconductive enhancement at subband-gap spectral regions.

On the other hand, the photoconductive degradation observed at $\sim 300 \mathrm{~K}$ can be connected with the creation of $D^{-}$centers. Here, as illustrated in Fig. $5, D^{-}$centers can be transformed from $D^{0}$ centers with the thermal energy at 300 K. ${ }^{1} D^{+}$centers may also be created simultaneously, while the existence is not essential here. $D^{-}$centers can act as recombination (or trapping) centers for holes due to Columbic attractive forces, and accordingly the photocurrent decreases with light soaking. These photoinduced charged defects are metastable, which can recover to the normal bonding structures with annealing at the glass-transition temperature. Note that this model is essentially the same as that proposed by Shimakawa et al. ${ }^{2,4}$

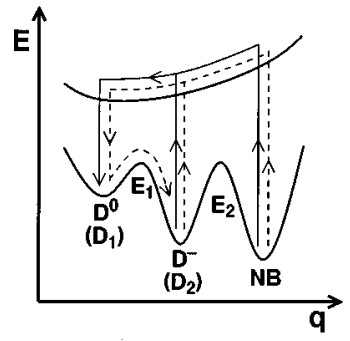

FIG. 5. The configuration-coordinate diagram of photoinduced changes. The solid and dashed lines with arrows represent the transformations responsible for light soaking at 13 and $300 \mathrm{~K}$.

\section{B. Intercluster interaction model}

The photoconductive enhancement and the degradation in chalcogenide glasses can also be understood without assuming the bond breaking. Ovshinsky pointed out the importance of interaction between lone-pair electrons of chalcogen atoms belonging to different segmental and/or fragmental clusters. ${ }^{25}$ Actually, Watanabe et al. ${ }^{26}$ demonstrated theoretically that lone-pair electrons of chalcogenide atoms interacting with each other through van der Waals forces can produce electrically neutral gap states in amorphous chalcogenides, which might be regarded as a kind of polaron model. ${ }^{1}$ In the following, the two quasistable states $D^{0}$ and $D^{-}$in Fig. 5 are replaced by the structures $D_{1}$ and $D_{2}$ illustrated in Fig. $6 .^{27}$

The photoinduced changes may be the following. In Fig. $6(\mathrm{a})$, there exists a normal bonding site, where the interaction between lone-pair electrons of chalcogen atoms is stronger, and accordingly the site can act as a hole trap. This is because the lone-pair electron states of chalcogen atoms form the top of the valence band, the width of which is governed by the interaction between the lone-pair electrons. $1,25,26$ When an electron-hole pair is excited by illumination, the hole can diffuse, leaving behind the electron which is less mobile. The hole may be captured by the hole trap, then it being trapped deeper through modifying the intercluster in-
NB

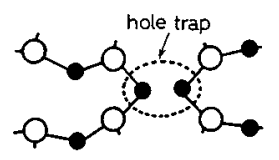

$D_{1}$

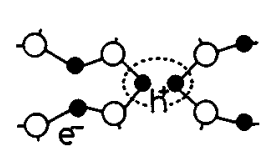

(a)

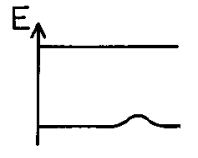

(b)

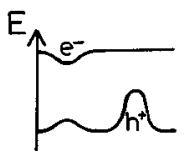

$\mathrm{D}_{2}$

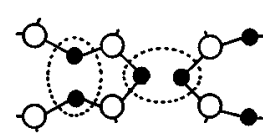

(c)

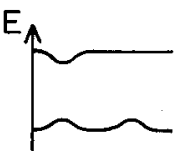

FIG. 6. Schematic illustrations of the intercluster interaction model. Atomic images are illustrated for $a-\mathrm{As}_{2} \mathrm{~S}_{3}$ with the corresponding energy bands. (a), (b), and (c) refer to the normal bonding structure, the light-soaked structure at low temperatures, and the light-soaked structure at room temperature, respectively. 
teraction. That is, lattice relaxation takes place. On the other hand, the electron is possibly self-trapped at the photoexcited site through deforming intracluster and intercluster structures. This site can provide localized states at the edges of the conduction and the valence band, the situation being illustrated in Fig. 6(b). Here, the trapped hole can cause the midgap absorption, since photoexcitation of an electron in the valence band to this hole is possible. Therefore, the photoconductive enhancement can occur. However, at room temperature, the thermal energy can excite the trapped electron, leaving the atomic structure unchanged. The electron can diffuse, and it may recombine with the trapped hole. Here, the atomic structure of the hole trap will partially be relaxed. In consequence, as shown in Fig. 6(c), the number of hole traps increases, which is considered to be the origin of the photoconductive degradation observed at room temperature.

\section{Material dependence}

In both models, the configuration diagram illustrated in Fig. 5 is commonly applicable, and accordingly it may be valuable to discuss the compositional trend using the diagram. First, the fact that the photoconductive enhancement at low temperatures is universally observed in chalcogenide glasses suggests the existence of $E_{1}$ which possesses a nearly constant height. Second, the result that the photoconductive degradation at $300 \mathrm{~K}$ is dependent on the materials can be reduced to the material-dependence of the barrier height of $E_{2}$. As the material is more metallic and/or the glasstransition temperature is lower, $E_{2}$ will be smaller. Then, even at room temperature no light-soaking effects can appear. Such situations most possibly apply to $a-\mathrm{As}_{2} \mathrm{Te}_{3}$ and $a$-Se.

The above qualitative idea can be developed to give quantitative evaluation of $E_{1}$ and $E_{2}$. The barrier heights can be estimated from measured lifetimes of the photoenhanced and photodegraded states through assuming thermally activated relaxation. ${ }^{1}$ That is,

$$
1 / \tau(T)=\Omega \exp \left(-E_{1,2} / k_{B} T\right),
$$

where $\tau$ is the typical lifetime of the photoenhanced or the photodegraded state, $\Omega$ is the vibrational frequency, which is approximated at $10^{13} \mathrm{~Hz},{ }^{1} E_{1,2}$ is the barrier height, and $T$ is the temperature. For instance, $E_{1}$ of $\mathrm{As}_{2} \mathrm{~S}_{3}$ can be estimated as follows. In $\mathrm{As}_{2} \mathrm{~S}_{3}$, the photoconductive enhanced state is held in stable at $100 \mathrm{~K}$ at least for $10 \mathrm{~min}$, while at $200 \mathrm{~K}$ the photoconductive enhancement does not appear. This observation means that for $D^{0}$ (or $\left.D 2\right) \tau(100 \mathrm{~K}) \geqslant 10^{3} \mathrm{sec}$ and $\tau(200 \mathrm{~K}) \leqslant 10^{0}$ sec. Then, solving Eq. (1), we obtain $E_{1}$ $=0.3-0.5 \mathrm{eV}$. In the same way, other barrier heights can be calculated; the results are listed in Table I. Since the photoconductive enhancement occurs in all the glassy materials at low temperatures, $E_{1}$ is almost the same in the materials, while $E_{2}$ seems to reflect the glass-transition temperatures.

\section{Comparison with (inverse) Staebler-Wronski effects}

Finally, it may be valuable to compare the present photoconductive enhancement with some observations in $a-\mathrm{Si}: \mathrm{H}$ with respect to the mechanisms. In $p$-type $a$-Si:H a phenom- enon called the inverse Staebler-Wronski effect is reported. ${ }^{28-32}$ Light soaking of $p$-type $a$-Si:H induces increases in the dark conductivity, ${ }^{28,30-32}$ and in some cases in the photoconductivity as well. ${ }^{29}$ In this respect, the phenomenon is similar to the present observations. However, the inverse Staebler-Wronski effect is interpreted to be manifestations of optical activation of oxide layers ${ }^{28}$ or doped atoms, ${ }^{29-32}$ which cannot be responsible for the present case.

Alternatively, the present photoconductive enhancement resembles the Staebler-Wronski effect in $a-\mathrm{Si}: \mathrm{H}$ in the respect that both accompany the midgap absorption., ${ }^{2,22}$ As demonstrated in the present study, however, in the chalcogenide glass at low temperatures the photocurrent increases with illumination, while in $a-\mathrm{Si}: \mathrm{H}$ at room temperature the photocurrent decreases with illumination. ${ }^{2}$ What causes this difference? A possible reason lies in the difference of carriertransport mechanisms in amorphous semiconductors at room and low temperatures. That is, at room temperature the transport occurs through extended states with multiple trapping and so forth, ${ }^{1}$ while at low temperatures it seems to be sustained by the quantum-mechanical hopping through localized band-tail states. ${ }^{18}$ Accordingly, the midgap states at room temperature are responsible for a reduction of the mobilitylifetime product of carriers, while at low temperatures the states can act only as carrier donors, not as recombination and/or trapping centers. If so, the photocurrent enhancement at subband-gap spectral regions could occur also in $a-\mathrm{Si}: \mathrm{H}$ at low temperatures. Actually, the result obtained using the constant-photocurrent method by Stradins and Fritzsche ${ }^{33}$ may imply such possibilities.

\section{CONCLUSIONS}

In chalcogenide glasses at low temperatures, the photocurrent increase at subband-gap spectral regions occurs with illumination. In contrast to the photoconductive degradation observed at room temperature, the photoconductive enhancement is universally observed in all the chalcogenide glasses investigated in the present study. On the other hand, in chalcogenide crystals, no light-soaking effects have been detected.

The photocurrent increase can be related with the photoinduced midgap absorption. That is, the midgap states are responsible for an increase in photoexcited carrier density. The carrier-transport mechanism is not possible influenced with light soaking. These ideas have been discussed with respect to the two microscopic models. The present study implies that the photoconductive enhancement seems to be inherent in amorphous semiconductors at low temperatures in which midgap states can be photoinduced.

\section{ACKNOWLEDGMENTS}

The authors would like to thank Dr. Kazunobu Tanaka for providing orpiment samples, and Dr. M. Yamaguchi and Dr. N. Yoshida for fruitful comments. The present work is partially supported with grants from the Ministry of Education and Yazaki Memorial Foundation. 
*Author to whom correspondence should be addressed: FAX: 81-011-716-6175. Electronic address: keiji@ hikari4.huap.hokudai.ac.jp

${ }^{1}$ N. F. Mott and E. A. Davis, Electronic Processes in NonCrystalline Materials, 2nd ed. (Clarendon, Oxford, 1979).

${ }^{2}$ K. Shimakawa, A. Kolobov, and S. R. Elliott, Adv. Phys. 44, 475 (1995).

${ }^{3}$ D. L. Staebler and C. R. Wronski, J. Appl. Phys. 51, 3262 (1980).

${ }^{4}$ K. Shimakawa, Phys. Rev. B 34, 8703 (1986).

${ }^{5}$ R. E. Johanson, H. Fritzsche, and A. Vomvas, J. Non-Cryst. Solids 114, 274 (1989).

${ }^{6}$ N. Toyosawa and K. Tanaka, Solid State Commun. 97, 623 (1996).

${ }^{7}$ S. R. Elliott, Physics of Amorphous Materials, 2nd ed. (Longman, Essex, 1990).

${ }^{8} \mathrm{Se}$ bulk glasses were likely to be partially crystallized, which influenced the electrical conductivity appreciably.

${ }^{9}$ This gives band-gap illumination for $\mathrm{As}_{2} \mathrm{~S}_{3}$.

${ }^{10}$ D. K. Biegelsen and R. A. Street, Phys. Rev. Lett. 44, 803 (1980).

${ }^{11}$ P. Kounavis and E. Mytilineou, Philos. Mag. Lett. 72, 117 (1995).

${ }^{12}$ K. Hayashi, Y. Hikida, K. Shimakawa, and S. R. Elliott, Philos. Mag. Lett. (to be published).

${ }^{13}$ J. T. Edmond, J. Non-Cryst. Solids 1, 39 (1968).

${ }^{14}$ J. I. Polanco, G. G. Roberts, and M. B. Myers, Philos. Mag. 25, 117 (1971).

${ }^{15}$ H. Moribe and M. Saji, Jpn. J. Appl. Phys. 13, 284 (1974).

${ }^{16}$ L. C. Bobb, K. Kramer, and H. H. Byer, J. Non-Cryst. Solids 21, 441 (1976).

${ }^{17}$ P. N. Banerjee, M. Datta, and D. L. Bhattacharya, Thin Solid Films 76, 179 (1981).

${ }^{18}$ H. Fritzsche, J. Non-Cryst. Solids 114, 1 (1989).

${ }^{19}$ H. Schlosser, J. Appl. Phys. 28, 512 (1957).

${ }^{20}$ B. T. Kolomiets, T. F. Mazets, Sh. M. Efendiev, and A. M. Andriesh, J. Non-Cryst. Solids 4, 45 (1970).

${ }^{21}$ S. W. Ing, Jr., J. H. Neyhart, and F. Schmidlin, J. Appl. Phys. 42, 696 (1971).
${ }^{22}$ S. G. Bishop, U. Strom, and P. C. Taylor, Phys. Rev. B 15, 2278 (1977).

${ }^{23}$ There may be a possibility that the excited state continuously relaxes to the $D^{0}$ state. However, since the photoluminescence of $\sim E_{g} / 2$ is characteristic of chalcogenide glasses at low temperatures (Ref. 1), we assume that the existence of the energy gap between the excited and the relaxed state is more plausible.

${ }^{24}$ In a previous study (Ref. 6), the existence of an energy barrier in the excited state is assumed. The barrier may be effective to suppress the transformation from normal bonding structures to $D^{0}$ defects at low temperatures. However, since the photocurrent enhanced state at low temperatures reduces to the photocurrent degraded state at room temperature (Fig. 2), the normal bonding structures may be broken even at low temperatures. Accordingly, the barrier is taken away in the present model.

${ }^{25}$ S. R. Ovshinsky and D. Adler, Contemp. Phys. 19, 109 (1978).

${ }^{26}$ Y. Watanabe, H. Kawazoe, and M. Yamane, Phys. Rev. B 38, 5677 (1988).

${ }^{27} \mathrm{We}$ should admit that, in some degree, that is a problem of terminology, since any atomic bond in the materials of interest cannot possess ideal covalency neither van der Waals in character.

${ }^{28}$ B. Aker and H. Fritzsche, J. Appl. Phys. 54, 6628 (1983).

${ }^{29}$ J. Jang, T. M. Kim, and J. K. Hyun, J. Non-Cryst. Solids 59\&60, 429 (1983).

${ }^{30}$ G. Ganguly, S. Ray, and A. K. Barua, Philos. Mag. B 54, 301 (1986).

${ }^{31}$ J. Jang, S. C. Park, S. C. Kim, and C. Lee, Appl. Phys. Lett. 51, 1804 (1987).

${ }^{32}$ M. Isomura, T. Kinoshita, and S. Tsuda, J. Non-Cryst. Solids 198-200, 453 (1996).

${ }^{33}$ P. Stradins and H. Fritzsche, Philos. Mag. B 69, 121 (1994).

${ }^{34}$ A. A. Andreev, B. T. Kolomiets, T. F. Mazets, A. L. Manukyan, and S. K. Pavlov, Sov. Phys. Solid State 18, 29 (1976).

${ }^{35}$ L. Tichý, H. Tichá, P. Nagels, E. Sleeckx, and R. Callaerts, Mater. Lett. 26, 279 (1996). 